\title{
The role of multiple SOS boxes upstream of the Mycobacterium tuberculosis lexA gene - identification of a novel DNA-damage- inducible gene
}

\author{
Edith M. Dullaghan, † Patricia C. Brooks and Elaine O. Davis
}

Division of Mycobacterial Research, National Institute for Medical Research, The Ridgeway, Mill Hill, London NW7 1AA, UK

\author{
Author for correspondence: Elaine O. Davis. Tel: +44 208959 3666. Fax: + 442089138528. \\ e-mail: edavis@nimr.mrc.ac.uk
}

\begin{abstract}
Four potential binding sites for LexA were identified upstream of the Mycobacterium tuberculosis lexA gene. A mutational analysis of these sites in a lexA-lacZ reporter construct revealed that only one of these SOS boxes was required for DNA-damage-mediated regulation of lexA expression. A novel DNA-damage-inducible gene, Rv2719c, was identified that was divergently transcribed relative to lexA; the other three SOS boxes were found to be involved in regulating expression of this novel mycobacterial-specific gene. The SOS boxes lay in the respective promoter regions of the genes that they regulated.
\end{abstract}

Keywords: LexA, SOS box, mycobacteria

\section{INTRODUCTION}

Bacteria respond to DNA damage by inducing various genes that are required for DNA repair or for DNA damage tolerance. The best characterized of these systems is the SOS response of Escherichia coli, in which over 40 genes are regulated by a common mechanism (Courcelle et al., 2001; Friedberg et al., 1995; Little \& Mount, 1982). At the heart of this regulatory mechanism is the repressor protein LexA, which binds to a particular sequence (CTGTatatatatACAG) (Friedberg et al., 1995), called the SOS box, upstream of the genes whose transcription it regulates (Brent \& Ptashne, 1981; Little et al., 1981). When DNA damage occurs, the recombination protein RecA binds to regions of singlestranded DNA resulting from processing of the damaged DNA or blockage of replication and forms nucleoprotein filaments (Sassanfar \& Roberts, 1990). In this activated form RecA stimulates the autocatalytic cleavage of LexA (Little, 1991), the fragments of which no longer bind to the SOS boxes (Bertrand-Burggraf et al., 1987), thus increasing transcription of the SOS genes.

The basic principles of this regulatory mechanism are found in many other species of bacteria, although the

\footnotetext{
†Present address: The University of British Columbia, The Research Centre, Faculty of Medicine, Dept of Paediatrics, 950 West 28th Avenue, Vancouver, BC, Canada V5Z 4H4.

Abbreviation: RACE, rapid amplification of CDNA ends.
}

DNA sequence of the LexA-binding site, or SOS box, varies. For example, in Bacillus subtilis the sequence CGAACRNRYGTTCG (Winterling et al., 1998) is recognized by the LexA homologue known as DinR. A related motif is used in mycobacteria; originally the motif GAACnnnnGTTC was shown to bind Mycobacterium tuberculosis LexA (Durbach et al., 1997; Movahedzadeh et al., 1997), whilst a more recent analysis of the requirements for LexA binding in vivo have revealed that the mycobacterial SOS box has the consensus sequence TCGAACnnnnGTTCGA (Davis et al., 2002).

The SOS box is generally positioned close to, and often overlaps, the promoter of the gene regulated by LexA binding to it. In E. coli there are examples of genes whose SOS boxes overlap the -35 sequence, are between the -35 and -10 sequences, overlap the -10 sequence or are downstream of the -10 sequence (Schnarr et al., 1991). Many genes have a single SOS box that is sufficient to confer regulation, and there are even some instances of divergently oriented genes each being regulated by a common SOS box (Courcelle et al., 2001). However, there are also a few examples of genes which possess two or even three SOS boxes. In E. coli the lexA gene has two SOS boxes separated by just $5 \mathrm{bp}$, one overlapping the -10 sequence and the other lying just downstream of the transcription start site (Brent \& Ptashne, 1981; Little et al., 1981). The recN gene of $E$. coli has three SOS boxes, one overlapping the -35 sequence, a second overlapping the -10 sequence and a 
third downstream of the transcription start site (Rostas et al., 1987).

In B. subtilis two motifs with homology to the then defined bacillus SOS box were initially identified upstream of recA, but a functional analysis of these sites revealed that only one of these is actually required for regulation of recA expression (Cheo et al., 1993). Similarly, there are three sites which bind the LexA homologue DinR upstream of $\operatorname{dinR}$ in B. subtilis (Haijema et al., 1996; Winterling et al., 1998), one of which overlaps the -35 sequence whilst the other two lie further upstream. However, just the site nearest to the gene is sufficient to confer DNA damage induction by the same factor as seen when all three are present (Haijema et al., 1996). There are also two SOS boxes upstream of $\operatorname{din} C$ in B. subtilis, one being between the -10 and -35 sequences whilst the other lies a few bases upstream of the -35 site. Although both of these sites bind DinR in vitro (Lovett et al., 1993; Miller et al., 1996; Winterling et al., 1998), their requirement for induction in vivo has not been reported.

Examination of the DNA sequence upstream of the $M$. tuberculosis lexA gene revealed several motifs with similarity to the mycobacterial SOS box. Here we investigate the roles they each play in regulating expression of lexA and of a divergent ORF, $R v 2719 c$, using transcriptional fusions to a lac $Z$ reporter gene.

\section{METHODS}

Bacterial strains and media. E. coli DH5 $\alpha$ was used for general cloning, whilst E. coli XL1Blue was used for site-directed mutagenesis (Sambrook et al., 1989). The mycobacterial strains used in this study were Mycobacterium smegmatis $\mathrm{mc}^{2} 155$ (Snapper et al., 1990) and M. tuberculosis H37Rv. E. coli was grown in L broth (Sambrook et al., 1989) and $M$. smegmatis and M. tuberculosis were grown in modified Dubos medium supplemented with albumen and $0 \cdot 2 \%$ (w/v) glycerol. E. coli and M. smegmatis were grown at $37^{\circ} \mathrm{C}$ with shaking and M. tuberculosis was grown at $37^{\circ} \mathrm{C}$ in a rolling incubator at 2 r.p.m. All procedures with live M. tuberculosis were carried out under Containment level 3 conditions. Antibiotics were added to the growth media as required: $100 \mu \mathrm{g}$ ampicillin $\mathrm{ml}^{-1}$ for E. coli; $50 \mu \mathrm{g} \mathrm{kanamycin} \mathrm{ml}^{-1}$ for $E$. coli; $20 \mu \mathrm{g}$ kanamycin $\mathrm{ml}^{-1}$ for mycobacteria.

Recombinant DNA techniques. Plasmid DNA was prepared using SNAP miniprep kits (Invitrogen) according to the manufacturer's instructions. Site-directed mutagenesis was performed as described in the instructions for the QuickChange Site-Directed Mutagenesis Kit (Stratagene). For other DNA manipulations, standard DNA protocols were followed (Sambrook et al., 1989). For each clone or mutant made, the sequences of the promoter region and the junctions to the vector were determined on an ABI PRISM 377 DNA sequencer using the ABI PRISM dRhodamine dye terminator cycle sequencing kit (PE Applied Biosystems).

Plasmid construction. A 587 bp PCR fragment containing the entire intergenic sequence upstream of $\operatorname{lex} A$ and extending into the coding sequence of a divergent ORF $(R v 2719 c)$ as well as that of lexA itself was generated using the primers GCTCTAGACGGCGCCAGCGAGGTCCATC and GCAAGCTTCGGGGGCGGTTCGGGTCAC. The bases in bold indicate restriction sites for $\mathrm{XbaI}$ and HindIII, respectively, which were added to facilitate cloning. Following its digestion with these restriction enzymes, the PCR product was cloned into the mycobacterial promoter-probe vector pEJ414 (Papavinasasundaram et al., 2001) to form a transcriptional fusion of the lexA promoter with the lac $Z$ reporter gene (pEMD8). Subsequently, a 315 bp PCR fragment was amplified using the primers CGAAGCTTATTGAGCGGATCGGGGGTAT and CGTCTAGAGAGGTGTCGTTGCTGTCGTTCA and similarly cloned into pEJ414 to form a transcriptional fusion of the $R v 2719$ c promoter with the lacZ reporter gene (pEJ544). This fragment included the entire intergenic sequence between the lexA and $R v 2719 c$ genes as well as extending into the coding sequence of $R v 2719 c$, but only overlapped the lexA mRNA rather than its coding sequence.

Introduction of clones into mycobacteria, and verification of insertion by PCR and sequencing. Published protocols were followed for preparing electrocompetent cells of M. smegmatis or M. tuberculosis (Papavinasasundaram et al., 1998) and for electroporation (Jacobs et al., 1991). After streaking out the resulting transformants, a preparation of total DNA suitable for PCR was isolated using InstaGene matrix (Bio$\mathrm{Rad}$ ) according to the manufacturer's instructions, except that we used more bacteria. The insert and junctions of each clone were isolated as a PCR product as described previously (Davis et al., 2002) and sequenced on an ABI PRISM 377 DNA sequencer using the ABI PRISM dRhodamine dye terminator cycle sequencing kit.

Induction conditions. To induce DNA damage in M. smegmatis transformants, mitomycin $\mathrm{C}$ was added to one aliquot of a culture at an $\mathrm{OD}_{600}$ value of between 0.5 and 0.6 to a final concentration of $0 \cdot 2 \mu \mathrm{g} \mathrm{ml} \mathrm{m}^{-1}$; the culture was then incubated for $5 \mathrm{~h}$. An equal volume of the same culture was also incubated for the same period of time without any addition, to provide an uninduced control. For M. tuberculosis, a similar procedure was followed except that cultures were induced at an $\mathrm{OD}_{600}$ value of between $0 \cdot 4$ and $0 \cdot 5$, and induction was for $24 \mathrm{~h}$. Following incubation in the presence or absence of the inducer, the bacteria were harvested, washed three times in $\mathrm{Z}$ buffer (Miller, 1972) without $\beta$-mercaptoethanol $\left(Z^{*}\right)$ and stored as a pellet at $-20^{\circ} \mathrm{C}$.

Preparation of cell-free extracts and $\beta$-galactosidase assays. Cell lysates were prepared, and protein and $\beta$-galactosidase assays were performed as described previously (Davis et al., 2002). In the case of M. tuberculosis lysates, the supernatants were filtered through a low-binding Durapore $0 \cdot 22 \mu \mathrm{M}$ membrane filter (Ultrafree-MC; Millipore) to eliminate any residual intact bacteria prior to assay. The specific activity, in units (mg protein $)^{-1}$, was calculated using the formula defined by Miller (1972).

Gel-shift assays. Gel-shift assays to analyse LexA binding to individual SOS boxes were performed as described previously (Brooks et al., 2001). The sequence of the oligonucleotide containing Box 3 was gtag TCactCatgtGTTCGAtatc, in which the bases matching the consensus SOS box sequence are shown in upper case. The complementary oligonucleotide was annealed with the aforementioned sequence and the resulting double-stranded probe was used in the assay. Binding to Box 3 was assayed alongside the other SOS boxes, which acted as controls.

Rapid amplification of cDNA ends (RACE) analysis. This procedure was carried out using a FirstChoice RLM-RACE kit (Ambion) according to the manufacturer's instructions but omitting the steps prior to ligation of the RACE adapter, which only apply to eukaryotic RNA. For RNA isolated from M. tuberculosis, two primers specific for $R v 2719 c$ were used for the nested PCR - an outer primer, RaceRv2719c (GACA- 
TCCCGACCCCGGTGCGGTGGTAGC), and an inner primer, XbaRv2719c (GGTCTAGAATTGAGCGGATCGGGGGTATGC), which included an $\mathrm{XbaI}$ site for cloning (shown in bold). For RNA isolated from M. smegmatis containing pEJ544 only the inner primer could be used, as the outer primer bound outside of the fragment cloned in pEJ544, so a semi-nested PCR was performed using XbaRv2719c and the two supplied primers that were complementary to the adapter. The cycle conditions for PCR were $94^{\circ} \mathrm{C}$ for $2 \mathrm{~min}$, followed by 35 cycles at $94^{\circ} \mathrm{C}$ for $30 \mathrm{~s}, 60^{\circ} \mathrm{C}$ for $30 \mathrm{~s}$ and $72{ }^{\circ} \mathrm{C}$ for $30 \mathrm{~s}$, with a final step at $72{ }^{\circ} \mathrm{C}$ for $7 \mathrm{~min}$. The final PCR

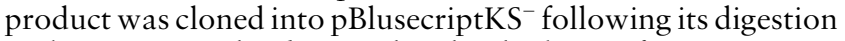
with $B a m \mathrm{HI}$ and $\mathrm{XbaI}$, and individual transformants were sequenced.

\section{RESULTS AND DISCUSSION}

\section{Identification of four SOS boxes upstream of lexA}

Examination of the sequence upstream of the $M$. tuberculosis lexA gene revealed the presence of four motifs with similarity to the recently defined mycobacterial SOS box (TCGAACnnnnGTTCGA). We have numbered these sites starting with that closest to the lexA coding region (Fig. 1). The individual motifs have different bases, and different numbers of bases, which differ from the consensus sequence. Thus, Box 1 (TCGAACacatGTTTGA) has a single mismatch, Box 2 (CCGAACatttGATCGA) and Box 4 (TCGCTCgcgtG-

CACCCGGCTGTCCGCACCAGCGGCTCCGCCGGCAA
CCGAGGTGTCGTTGCTGTCGTTCATGGCAATGAAT
lexA GTAGTCGCATTCGCACCAAGAATCAAACATGTGTT

CGACAGGCGTGTTCCGGTCGCGGGAGGGCACGAGG

\section{AGTGGCTGGGCCTTCGCGCCGGAAGTCCGGCTCCA}

ACCGAAACGTGTCGGTGGTGTGGTCTACCGTTTTG
$\underset{R}{\rightarrow} 2719 c 2$
BGCGTGCGACACGCTTCGATCAAATGTTCGGATAT
Box 3
CGAACACATGAGTGACTACAGTGTCGAACACGCGA

Box 4

GCGATTACCGTCTGAGTTGGAGGAGCGAACATG

Fig. 1. Sequence upstream of the $M$. tuberculosis lexA gene The sequence is shown in the opposite direction to lexA, beginning at the predicted start codon of LexA and continuing to that of $R v 2719 c$, which is transcribed divergently from lexA. Motifs resembling SOS boxes are boxed and numbered 1-4; the predicted initiation codons for each gene are underlined. The transcriptional start site of lexA, determined previously (Movahedzadeh et al., 1997), is indicated by the left-facing arrow. Two possible transcriptional start sites for Rv2719c, determined here by RACE analysis, are indicated by the rightfacing arrows; the majority of clones were consistent with the solid arrow, whilst a longer product observed in two clones suggested the possibility of initiation at the dotted arrow.
TTCGA) each have two mismatches and Box 3 (TCACTCatgtGTTCGA) has three mismatches from the consensus (the mismatches being highlighted in bold). Intriguingly, inspection of the Mycobacterium leprae sequence (Cole et al., 2001) reveals the presence of a motif with only a single mismatch (TCGATCatatGTTCGA) in a position identical to Box 3. Box 2 and Box 3 are separated by only $3 \mathrm{bp}$, there are just 8 bp between Box 3 and Box 4, and Box 1 and Box 2 are separated by 117 bp (Fig. 1). Thus, the first bases of Boxes 2, 3 and 4 are located 181, 200 and 224 bp, respectively, upstream of the transcription start point of lexA, whilst Box 1 lies in the region of the lex $A$ promoter -35 element.

Three of the sites are the same as three of those identified using the earlier, shorter SOS consensus sequence (Brooks et al., 2001) whilst the remaining site (Box 3) differs. In the study of Brooks et al. (2001) it was shown that two of these sites (Boxes 1 and 4) bound LexA under physiologically relevant conditions, but no binding to Box 2 was detected. Interestingly, LexA also bound to Box 3 as assessed by gel shift under the same conditions (data not shown), although Box 3 contained more mismatches than the other sites. Therefore, it remained possible that LexA would bind to Box 2 in its native context when Boxes 3 and 4 were present very close to Box 2.

\section{The roles of each of the four SOS boxes in regulating lexA expression}

The roles of each of the four SOS boxes in regulating lexA expression were investigated by a mutagenic approach using a fusion of the lexA promoter, which included the upstream region containing all of the SOS boxes, to the reporter gene lac $Z$ in an integrating plasmid (pEMD8). Mutations were made which eliminated LexA binding to each SOS box individually, by changing the core of the sequence to TCCAnnnnTGGA. Box 2 was included in this analysis, even though it had failed to bind LexA in the gel-shift assay, to investigate whether it played a role when in the presence of the other SOS boxes.

The wild-type and mutated constructs were introduced into M. smegmatis $\mathrm{mc}^{2} 155$ and expression of the lac $Z$ reporter gene was assessed following growth of the strains in both the absence and presence of the DNAdamaging agent mitomycin $\mathrm{C}\left(0 \cdot 2 \mu \mathrm{g} \mathrm{ml}^{-1}\right)$. As expected, the wild-type sequence containing the lex $A$ promoter and all four SOS boxes conferred DNA-damage-inducible expression, with an induction ratio of $5 \cdot 4$ (Fig. 2a). Interestingly, when Box 1 alone was eliminated expression was no longer DNA-damage-inducible, with the induction ratio falling to $0 \cdot 5$ (Fig. 2a). Normally when a repressor protein is prevented from binding to its regulatory site the result is constitutive expression at around the level seen for the induced wild-type. However, the level of expression seen for this Box 1 mutation was substantially lower than the induced wild-type level; this is presumably because the mutation introduced also has an effect on promoter activity. 
(a)
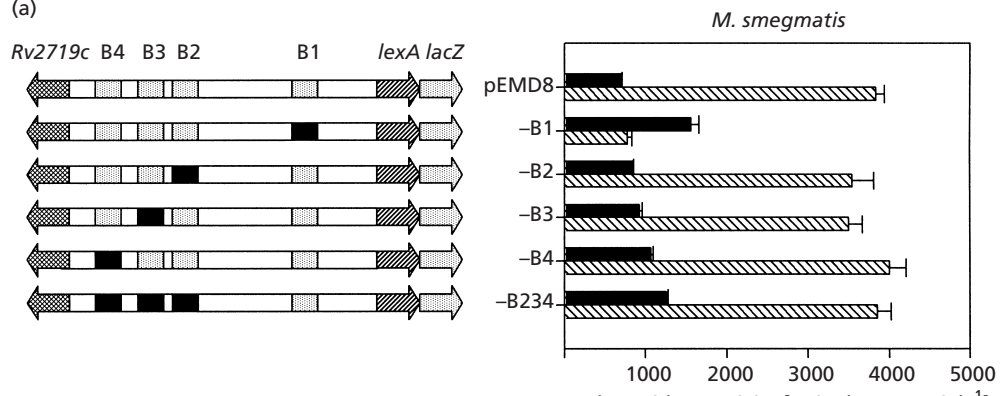

(b)

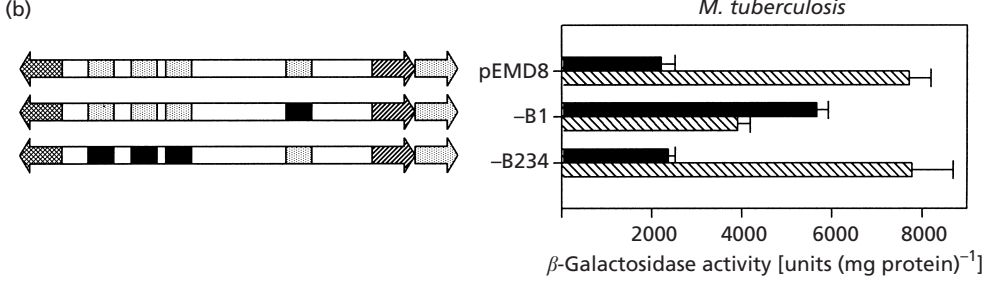

Fig. 2. DNA damage induction of the lexA promoter with and without mutations in the SOS boxes in (a) $M$. smegmatis and (b) $M$. tuberculosis. The lexA promoter was transcriptionally fused to a lac $Z$ reporter gene and $\beta$-galactosidase activity for each construct was determined following incubation in the presence (hatched bars) or absence (solid bars) of mitomycin C $\left(0.2 \mu \mathrm{g} \mathrm{ml}^{-1}\right)$. The graph shows the mean values obtained from duplicate assays of at least three independent inductions, with the error bars showing the SD. The SOS boxes that were mutated are shown as solid blocks in the schematic, where B1 refers to Box 1, B2 refers to Box 2, etc.
In contrast, constructs containing a Box 2, Box 3 or Box 4 knockout mutation individually showed similar patterns of expression to the wild-type (Fig. 2a). To ascertain whether this might be because one of these boxes could compensate for a mutation in another, we introduced mutations into each of Boxes 2, 3 and 4 simultaneously and assessed the effect of these changes on expression. Once again, expression remained clearly inducible. A small increase in the uninduced level of expression was observed, suggesting that the presence of LexA at these sites may play a minor role in downregulating expression from the lexA promoter. However, taken together the results from these experiments clearly show that induction is determined by binding of LexA to Box 1.

To confirm that this was also the case in M. tuberculosis itself, the constructs containing the wild-type sequence, the Box 1 mutation and the combined Boxes 2, 3 and 4 mutations were introduced into M. tuberculosis H37Rv and expression was assessed following growth of the recombinant strains in the absence and presence of mitomycin C. The absolute expression levels in these strains were about twofold higher than that seen in $M$. smegmatis, but the pattern of expression from the mutated constructs was the same. Thus, the mutation in Box 1 eliminated induction, whilst the strain carrying the combined mutations in Boxes 2, 3 and 4 showed expression equivalent to that of the wild-type (Fig. $2 b$ ).

\section{Could the other SOS boxes be regulating expression of a divergent gene?}

Upstream from the M. tuberculosis lexA gene there is a divergent gene of unknown function termed $R v 2719 \mathrm{c}$ (Cole et al., 1998). All four of the SOS boxes under study here are also upstream of this gene. As Boxes 2, 3 and 4 do not appear to have a role in regulating the expression of $\operatorname{lex} A$, it was conceivable that they might regulate the expression of $R v 2719 c$ instead. To investigate this possibility, we first determined whether the expression of $R v 2719 c$ was DNA-damage-inducible. A transcriptional lac Z fusion of the $R v 2719 c$ promoter and upstream region, containing all four SOS boxes, was made by cloning part of the fragment used above for the lex $A$ reporter construct in the opposite orientation. Assays of lac $Z$ reporter gene expression in $M$. smegmatis with and without treatment with the DNA-damaging agent mitomycin $C\left(0 \cdot 2 \mu \mathrm{g} \mathrm{ml}^{-1}\right)$ revealed that the expression of this ORF was indeed DNA-damage-inducible, with an induction ratio of 4.0 (Fig. 3a). When the corresponding constructs containing mutations which eliminated LexA binding to each SOS box were examined individually, the greatest reduction in the induction ratio, to $1 \cdot 4$, was seen with the Box 2 mutation, with smaller changes seen with the Box 3 or Box 4 mutations and very little effect seen with the Box 1 mutation. As no single SOS box knockout resulted in constitutive expression, we went on to examine various combinations of mutations. When any two of Boxes 2, 3 and 4 were mutated simultaneously the induction ratio was reduced to ca. $1 \cdot 3$, whilst the triple knockout of Boxes 2, 3 and 4 resulted in completely constitutive expression, with an uninduced level similar to that of the wild-type induced level (Fig. 3a). Thus, Boxes 2, 3 and 4 are all involved in regulating the expression of $R v 2719 c$.

Again, this conclusion was confirmed in M. tuberculosis using the constructs containing the wild-type sequence, the Box 1 mutation and the combined Boxes 2, 3 and 4 mutations. In the first place, this analysis confirmed that expression of $R v 2719 c$ was indeed DNA-damage-inducible in M. tuberculosis. Although the induction ratio was lower than that seen in M. smegmatis, the absolute expression level was higher than that of M. smegmatis, as seen with the lexA promoter. Significantly, the pattern of expression from the mutated constructs was the same as in M. smegmatis. Thus, the mutation in Box 1 remained inducible, whilst the combined mutations in Boxes 2, 3 and 4 resulted in constitutive expression (Fig. 3b). 

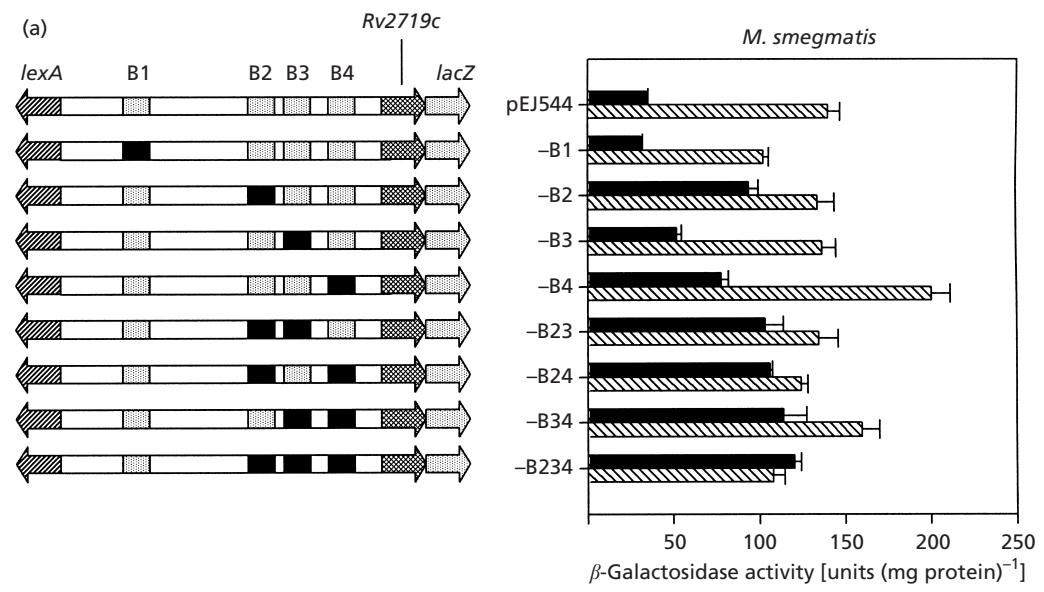

(b)

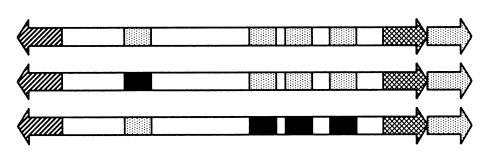

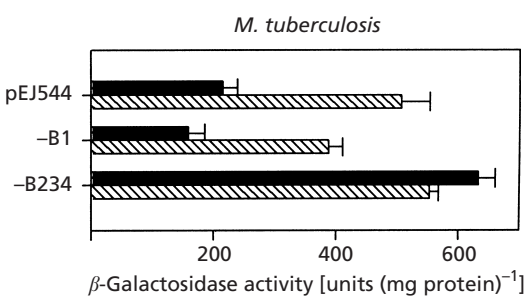

Fig. 3. DNA-damage induction of the Rv2719c promoter with and without mutations in the SOS boxes in (a) $M$. smegmatis and (b) $M$. tuberculosis. The Rv2719c promoter was transcriptionally fused to a lac $Z$ reporter gene and $\beta$ galactosidase activity for each construct was determined following incubation in the presence (hatched bars) or absence (solid bars) of mitomycin $\mathrm{C}\left(0.2 \mu \mathrm{g} \mathrm{ml}^{-1}\right)$. The graph shows the mean values obtained from duplicate assays of at least three independent inductions, with the error bars showing the SD. The SOS boxes that were mutated are shown as solid blocks in the schematic, where B1 refers to Box 1, B2 refers to Box 2, etc.
It has previously been reported that some genes in $M$. tuberculosis are DNA-damage-inducible even though they lack binding sites for LexA (Brooks et al., 2001), suggesting that an alternative mechanism of induction exists. Nevertheless, mutation of the SOS boxes to prevent LexA binding eliminated DNA damage induction of $l e x A$ and $R v 2719 c$, indicating that these genes are regulated by LexA in M. tuberculosis.

\section{The SOS boxes lie in the respective promoter regions of the genes that they regulate}

The specific effects of mutating Box 1 on lex $A$ expression and of mutating Boxes 2, 3 and 4 on $R v 2719 c$ expression could be explained by the locations of these boxes relative to the promoters for both of these genes. The transcriptional start site of $\operatorname{lex} A$ has been determined previously (Movahedzadeh et al., 1997), and indicates that Box 1 overlaps or lies close to the -35 site of the lexA promoter. We would predict that the promoter elements for $R v 2719 c$ would lie around or between Boxes 2, 3 and 4. Such an arrangement would explain why Box 1 regulates expression of lexA only and Boxes 2, 3 and 4 regulate expression of $R v 2719 c$ only.

To test the hypothesis that the promoter elements for $R v 2719 c$ lie around or between Boxes 2, 3 and 4, we attempted to determine the transcriptional start site of $R v 2719 c$. This was done by RACE using RNA extracted from M. tuberculosis and from M. smegmatis bearing the $R v 2719 c-l a c Z$ fusion. The majority of clones analysed were consistent with the transcriptional start site being an A located $36 \mathrm{bp}$ upstream of the predicted initiation codon (Fig. 1) in M. tuberculosis itself and when the M. tuberculosis promoter was being expressed in M. smegmatis. This A is situated within Box 4, and inspection of the sequence upstream from this point revealed a motif (TACAGT) with similarity to the $E$. coli $\sigma^{70}-10$ consensus sequence positioned between Box 3 and Box 4. A further motif (CGGATA) bearing some resemblance to the corresponding -35 consensus is located upstream of this, with a spacing of $17 \mathrm{bp}$ from the putative -10 motif; this putative -35 motif partially overlaps Box 2. However, the possibility of transcription in $M$. tuberculosis starting further upstream than this was suggested by a longer product observed in two instances, which would correlate with initiation at a G 97 bp upstream of the coding sequence. This $G$ is located upstream of all three of the SOS boxes that regulate the expression of $R v 2719 c$. In either case, Boxes 2, 3 and 4 are appropriately positioned to regulate $R v 2719 c$ whereas Box 1 is not, in accordance with the results described above.

Analogous to our findings for M. tuberculosis, it may be that the two SOS boxes further upstream of $\operatorname{din} R$ in $B$. subtilis are regulating a divergent ORF. Indeed, a divergent ORF termed $y n e A$ is found upstream of $d i n R$. There are good matches to -35 and -10 motifs a similar distance upstream of $y n e A$ to those reported for $\operatorname{din} R$ and the central SOS box of the three is located between these motifs, a typical position for a LexAbinding site.

\section{$R v 2719 c$ homologues appear to be regulated similarly in other mycobacteria}

No homologues of $R v 2719 c$ were found by searching the general sequence databases, but homologues of this gene were found in other mycobacterial species for which 

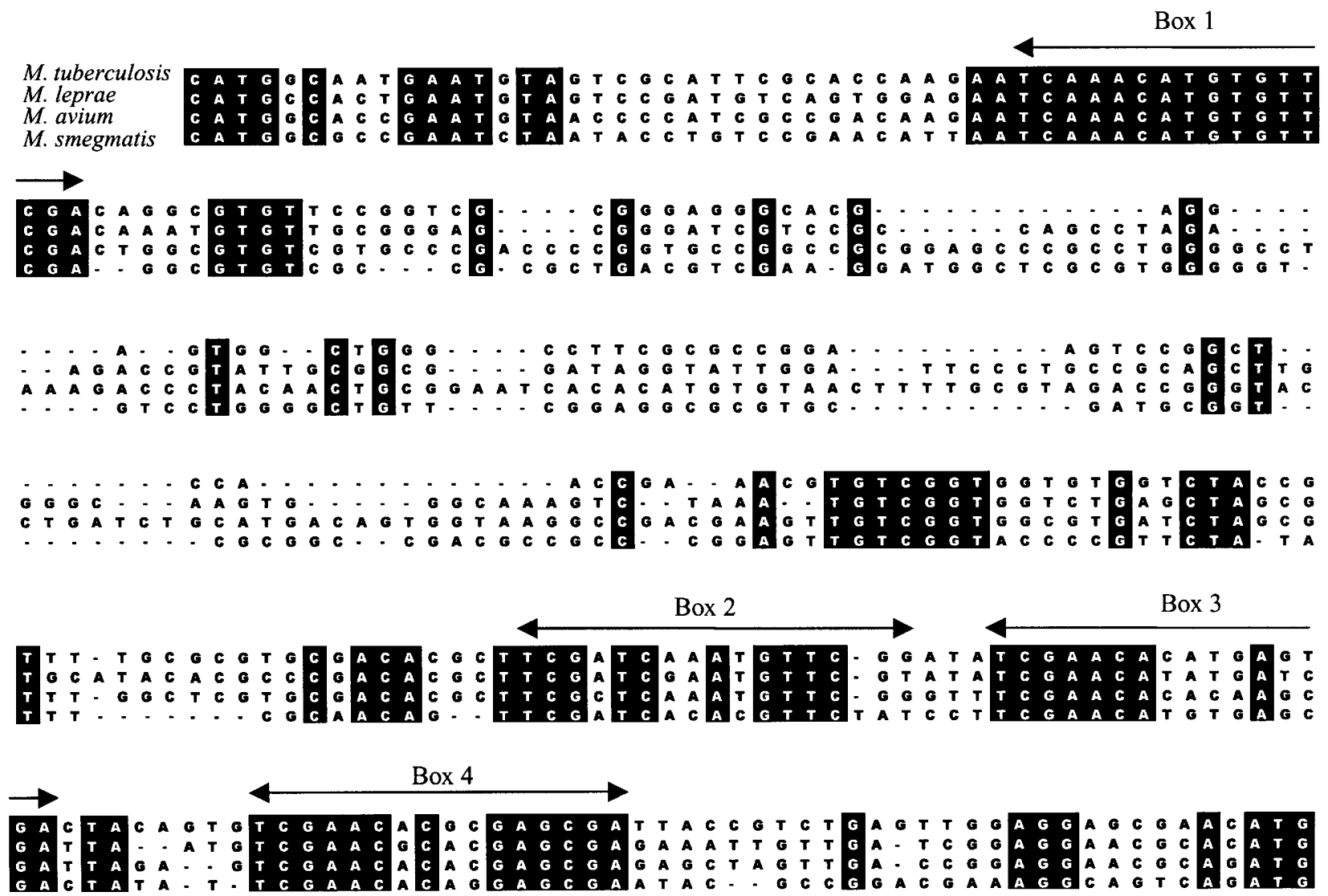

Fig. 4. Alignment of the intergenic regions between lexA and Rv2719c from $M$. tuberculosis, $M$. leprae, $M$. avium and $M$. smegmatis. The sequence is shown from two bases beyond the lexA transcriptional start site, which is 57 bp before the predicted start codon (see Fig. 1), to the predicted initiation codon of Rv2719c. Identical bases are highlighted and the four SOS boxes are marked.

genomic sequence information is available in a readily searchable form, namely M. leprae, Mycobacterium avium and M. smegmatis (TBLASTN scores of $8 \times 10^{-34}$, $1.6 \times 10^{-39}$ and $1 \times 10^{-31}$, respectively). Thus, this ORF appears to represent a mycobacterial-specific gene. Not only was a homologue present in each of these mycobacterial species but it was also located in the same relative position in the genome, i.e. upstream of lexA and in the opposite orientation to it. Significantly, there are blocks of sequence identity amongst $M$. tuberculosis, M. leprae, M. avium and M. smegmatis in the intergenic region between $\operatorname{lex} A$ and $R v 2719 c$, and all four SOS boxes are conserved in all four species (Fig. 4). Thus, the regulation of lexA by a single SOS box and of $R v 2719 \mathrm{c}$ homologues by three SOS boxes is common amongst mycobacteria. It would appear that having multiple copies of the SOS box can compensate for the presence of multiple mismatches in the individual motifs.

The function of $R v 2719 c$ is unknown. However, the database generated by a recent novel approach to predicting protein functional classes from sequence data (King et al., 2000) suggests that the product of $R v 2719 c$ is involved in cell-envelope macromolecule metabolism. The DNA-damage-inducible gene $\operatorname{din} C$ of $B$. subtilis is also known as $\operatorname{tag} C$ as it was thought to play a role in polyglycerol phosphate teichoic acid biosynthesis, although whether this is the case remains unresolved (Mauel et al., 1994).

In conclusion, the results of the present study suggest that some DNA-damage-inducible genes in M. tuberculosis may be regulated by LexA binding to multiple sites with two or more mismatches from the consensus sequence, rather than to a single site with up to one mismatch. This may allow a more graded response, depending on the degree of DNA damage. However, it also complicates the identification of further LexAregulated genes by motif identification.

\section{ACKNOWLEDGEMENTS}

We would like to thank Dr M. J. Colston for critical reading of the manuscript.

\section{REFERENCES}

Bertrand-Burggraf, E., Hurstel, S., Daune, M. \& Schnarr, M. (1987). Promoter properties and negative regulation of the uvrA gene by the LexA repressor and its amino-terminal DNA binding domain. J Mol Biol 193, 293-302.

Brent, R. \& Ptashne, M. (1981). Mechanism of action of the lexA gene product. Proc Natl Acad Sci U S A 78, 4204-4208. 
Brooks, P. C., Movahedzadeh, F. \& Davis, E. O. (2001). Identification of some DNA damage-inducible genes of Mycobacterium tuberculosis: apparent lack of correlation with LexA binding. J Bacteriol 183, 4459-4467.

Cheo, D. L., Bayles, K. W. \& Yasbin, R. E. (1993). Elucidation of regulatory elements that control damage induction and competence induction of the Bacillus subtilis SOS system. J Bacteriol 175, 5907-5915.

Cole, S. T., Brosch, R., Parkhill, J. \& 39 other authors (1998). Deciphering the biology of Mycobacterium tuberculosis from the complete genome sequence. Nature 393, 537-544.

Cole, S. T., Eiglmeier, K., Parkhill, J. \& 41 other authors (2001). Massive gene decay in the leprosy bacillus. Nature 409, 10071011.

Courcelle, J., Khodursky, A., Peter, B., Brown, P. O. \& Hanawalt, P. C. (2001). Comparative gene expression profiles following UV exposure in wild-type and SOS-deficient Escherichia coli. Genetics 158, 41-64.

Davis, E. O., Dullaghan, E. M. \& Rand, L. (2002). Definition of the mycobacterial SOS box and use to identify LexA-regulated genes in Mycobacterium tuberculosis. J Bacteriol 184, 3287-3295.

Durbach, S. I., Andersen, S. J. \& Mizrahi, V. (1997). SOS induction in mycobacteria: analysis of the DNA-binding activity of a LexAlike repressor and its role in DNA damage induction of the recA gene from Mycobacterium smegmatis. Mol Microbiol 26, 643653.

Friedberg, E., Walker, G. \& Siede, W. (1995). DNA Repair and Mutagenesis. Washington, DC: American Society for Microbiology.

Haijema, B. J., van Sinderen, D., Winterling, K., Kooistra, J., Venema, G. \& Hamoen, L. W. (1996). Regulated expression of the $\operatorname{dinR}$ and recA genes during competence development and SOS induction in Bacillus subtilis. Mol Microbiol 22, 75-85.

Jacobs, W. R., Jr, Kalpana, G. V., Cirillo, J. D., Pascopella, L., Snapper, S. B., Udani, R. A., Jones, W., Barletta, R. G. \& Bloom, B. R. (1991). Genetic systems for mycobacteria. Methods Enzymol 204, 537-555.

King, R. D., Karwath, A., Clare, A. \& Dehaspe, L. (2000). Accurate prediction of protein functional class from sequence in the Mycobacterium tuberculosis and Escherichia coli genomes using data mining. Yeast 17, 283-293.

Little, J. W. (1991). Mechanism of specific LexA cleavage: autodigestion and the role of RecA coprotease. Biochimie 73, 411-421.

Little, J. W. \& Mount, D. W. (1982). The SOS regulatory system of Escherichia coli. Cell 29, 11-22.

Little, J. W., Mount, D. W. \& Yanisch-Perron, C. R. (1981). Purified lexA protein is a repressor of the recA and lexA genes. Proc Natl Acad Sci U S A 78, 4199-4203.
Lovett, C. M., Jr, Cho, K. C. \& O'Gara, T. M. (1993). Purification of an SOS repressor from Bacillus subtilis. J Bacteriol 175, 68426849.

Mauel, C., Young, M., Monsutti-Grecescu, A., Marriott, S. A. \& Karamata, D. (1994). Analysis of Bacillus subtilis tag gene expression using transcriptional fusions. Microbiology 140, 2279-2288.

Miller, J. (1972). Experiments in Molecular Genetics. Cold Spring Harbor, NY: Cold Spring Harbor Laboratory.

Miller, M. C., Resnick, J. B., Smith, B. T. \& Lovett, C. M., Jr (1996). The Bacillus subtilis dinR gene codes for the analogue of Escherichia coli LexA. Purification and characterization of the DinR protein. J Biol Chem 271, 33502-33508.

Movahedzadeh, F., Colston, M. J. \& Davis, E. O. (1997). Characterization of Mycobacterium tuberculosis LexA: recognition of a Cheo (Bacillus-type SOS) box. Microbiology 143, 929-936.

Papavinasasundaram, K. G., Colston, M. J. \& Davis, E. O. (1998). Construction and complementation of a recA deletion mutant of Mycobacterium smegmatis reveals that the intein in Mycobacterium tuberculosis recA does not affect RecA function. Mol Microbiol 30, 525-534.

Papavinasasundaram, K. G., Anderson, C., Brooks, P. C., Thomas, N. A., Movahedzadeh, F., Jenner, P. J., Colston, M. J. \& Davis, E. O. (2001). Slow induction of RecA by DNA damage in Mycobacterium tuberculosis. Microbiology 147, 3271-3279.

Rostas, K., Morton, S. J., Picksley, S. M. \& Lloyd, R. G. (1987). Nucleotide sequence and LexA regulation of the Escherichia coli recN gene. Nucleic Acids Res 15, 5041-5049.

Sambrook, J., Fritsch, E. F. \& Maniatis, T. (1989). Molecular Cloning: a Laboratory Manual, 2nd edn. Cold Spring Harbor, NY: Cold Spring Harbor Laboratory.

Sassanfar, M. \& Roberts, J. W. (1990). Nature of the SOS-inducing signal in Escherichia coli. The involvement of DNA replication. J Mol Biol 212, 79-96.

Schnarr, M., Oertel-Buchheit, P., Kazmaier, M. \& GrangerSchnarr, M. (1991). DNA binding properties of the LexA repressor. Biochimie 73, 423-431.

Snapper, S. B., Melton, R. E., Mustafa, S., Kieser, T. \& Jacobs, W. R., Jr (1990). Isolation and characterization of efficient plasmid transformation mutants of Mycobacterium smegmatis. Mol Microbiol 4, 1911-1919.

Winterling, K. W., Chafin, D., Hayes, J. J., Sun, J., Levine, A. S., Yasbin, R. E. \& Woodgate, R. (1998). The Bacillus subtilis DinR binding site: redefinition of the consensus sequence. J Bacteriol 180, 2201-2211.

Received 17 May 2002; revised 24 June 2002; accepted 16 August 2002. 